\title{
Metodología para el Modelado y simulación de pruebas de fatiga en álabes de aerogeneradores de baja potencia
}

\section{Modeling and simulation of fatigue tests on blades of low-powered wind turbines}

RUIZ-NUCAMENDI, Nelson Octavioł*, ROBLES-OCAMPO, Jose Billerman, SEVILLACAMACHO, Perla Yasmin y MORALES-ALIAS, Luis Alberto \author{
Chiapas \\ ID 1 ${ }^{\mathrm{er}}$ Autor: Nelson Octavio, Ruiz-Nucamendi / ORC ID: 0000-0002-2437-7676 \\ ID $1^{\text {er }}$ Coautor: Jose Billerman, Robles-Ocampo / ORC ID: 0000-0002-0094-8710 \\ ID $2^{\text {do }}$ Coautor: Perla Yasmin, Sevilla-Camacho / ORC ID: 0000-0002-9702-1929 \\ ID $3^{\text {er }}$ Coautor Luis Alberto, Morales-Alias / ORC ID: 0000-0003-0978-0595
}

Universidad Politécnica de Chiapas, Carretera Tuxtla-Portillo Zaragoza Villaflores KM.21+500, Las Brisas, 29150, 29150

DOI: $10.35429 /$ JSL.2019.20.6.23.32

Recibido 23 Junio, 2019; Aceptado 30 Septiembre, 2019

\section{Resuemen}

En este artículo se presenta el diseño, simulación y análisis de fatiga de varios perfiles aerodinámicos usados en aerogeneradores de baja potencia. Para ello, se desarrolla el modelo de un álabe de un aerogenerador de eje horizontal de $5 \mathrm{~kW}$ de potencia nominal. El análisis de los coeficientes de sustentación, arrastre y potencia de los perfiles aerodinámicos se realizó con el software XFLR5. La metodología que se empleó para el diseño del álabe, está basada en el método de interacciones y convergencia llamado BEM. Asimismo, para simular la parte estructural y aerodinámica del elemento, se utilizó el programa QBlade. Con el objetivo principal de garantizar que se alcanzan los factores de seguridad para la fatiga mencionados en la norma IEC 61400, se aplicó el Modelo de Cargas Simplificado. Se obtuvo como resultados el valor máximo de fatiga de $21,421.66 \mathrm{~N}$ y el valor máximo de momento en sentido flapwise de $698.41 \mathrm{Nm}$.

Fatiga, Álabe, Simulación en QBlade

\begin{abstract}
This article presents the design, simulation and fatigue analysis of various aerodynamic profiles used in low power wind turbines. For this purpose, the model of a blade of a horizontal axis wind turbine with a nominal power of $5 \mathrm{~kW}$ is developed. The analysis of the lift, drag and power coefficients of the aerodynamic profiles was carried out with the XFLR5 software. The methodology used for the blade design is based on the interactions and convergence method called BEM. Also, to simulate the structural and aerodynamic part of the element, the QBlade program was used. With the main objective of ensuring that the fatigue safety factors mentioned in the IEC 61400 standard are achieved, the Simplified Load Model was applied. The maximum fatigue value of $21,421.66 \mathrm{~N}$ and the maximum flapwise moment value of $698.41 \mathrm{Nm}$ were obtained.
\end{abstract}

Fatigue, Blade, QBlade simulation

Citación: RUIZ-NUCAMENDI, Nelson Octavio, ROBLES-OCAMPO, Jose Billerman, SEVILLA-CAMACHO, Perla Yasmin y MORALES-ALIAS, Luis Alberto. Metodología para el Modelado y simulación de pruebas de fatiga en álabes de aerogeneradores de baja potencia. Revista de Simulación y Laboratorio. 2019, 6-20: 23-32

\footnotetext{
*Correspondencia al Autor (Correo electrónico: noctavio_ruiz@ hotmail.com)

$\dagger$ Investigador contribuyendo como primer Autor
} 


\section{Nomenclatura}

\section{Nomenclatura}

Símbolo Descripción

Área de sección transversal

Unidad

Factor de inducción axial

Factor de inducción tangencial

Número de aspas

Coeficiente de potencia

Coeficiente de empuje

Coeficiente de sustentación

Coeficiente de momento

Coeficiente normal

Coeficiente tangencial

Cuerda

Torque

Aceleración de la gravedad

Factor de forma Weibull

$m_{B} \quad$ Masa del aspa

$m^{\prime} \quad$ Flujo másico

$P \quad$ Potencia

$Q_{\text {diseño }}$ Par de torsión de diseño del rotor

$R \quad$ Radio

$R_{i} \quad$ Posición radial

$T \quad$ Fuerza de Empuje

$V_{m} \quad$ Velocidad media del viento

$V_{\text {rel }} \quad$ Velocidad relativa

$\alpha \quad$ Ángulo de ataque

$\alpha_{\text {stall }}$ Ángulo de ataque con pérdida

aerodinámica

$\beta \quad$ Ángulo de torsión

$\theta \quad$ Ángulo de pitch o paso

$\theta_{T} \quad$ Ángulo de torsión

$\lambda \quad$ Relación de velocidad de la

punta del aspa

Viscosidad

Viscosidad cinemática

Densidad del aire

Solidez

Ángulo de flujo

Función Gamma

\section{Introducción}

La energía eólica es una de las fuentes de energía que más destacada entre las energías renovables, por la disminución de los precios de su tecnología. Aunque, los aerogeneradores han evolucionado fuertemente, aun no se ha logrado obtener el sistema ideal debido a la variabilidad de su fuente motriz. En México, el desarrollo de aerogeneradores se ha encaminado a la fabricación de prototipos de baja potencia, sin embargo, no se tienen registros de unidades vendidas. Bajo este contexto, existe la necesidad de desarrollar tecnología propia que permita la independencia tecnológica del extranjero y el aumento de la competitividad a nivel nacional e internacional de la industria eólica en el área de diseño y fabricación de aerogeneradores de baja potencia.
Durante el funcionamiento de un aerogenerador el álabe es el elemento encargado de convertir la energía cinética y presión del aire en energía mecánica. Por ello, la variedad y magnitud de cargas a las que se ven sometidos los álabes requieren de un diseño estructural y aerodinámico que respondan a la complejidad del elemento. La norma IEC 61400-2 (International Electrotechnical Comission, 2013) presenta los requerimientos de diseño necesarios para aerogeneradores de baja potencia.

Este trabajo presenta la metodología del modelado y simulación de pruebas de fatiga un álabe para aerogeneradores de baja potencia aplicando la norma IEC 61400 con ayuda de los softwares XFLR5 y QBlade. Para el desarrollo del trabajo se proponen 4 perfiles aerodinámicos (A18, BW3, NACA4421, NACA4424). Estos perfiles se analizan siguiendo la metodología para la evaluación de sus características de acuerdo a las necesidades del diseño y potencia.

\section{Metodología}

En esta sección se aborda la metodología propuesta para el diseño de un álabe y simulación de pruebas de fatiga a ser utilizada en aerogeneradores de baja potencia. Se ha estructurado en cuatro partes: la primera parte está dedicada a la selección del perfil aerodinámico, a través de la aplicación de criterios de diseño; la segunda parte presenta el dimensionamiento del álabe basada en el método BEM; la tercera parte muestra el modelado y la simulación aerodinámica realizada con el programa QBlade; y la cuarta parte muestra la aplicación del modelo de cargas simplificado (MCS) para el análisis de fatiga. En la Figura 1 se muestra el diagrama de la metodología del diseño del álabe. 


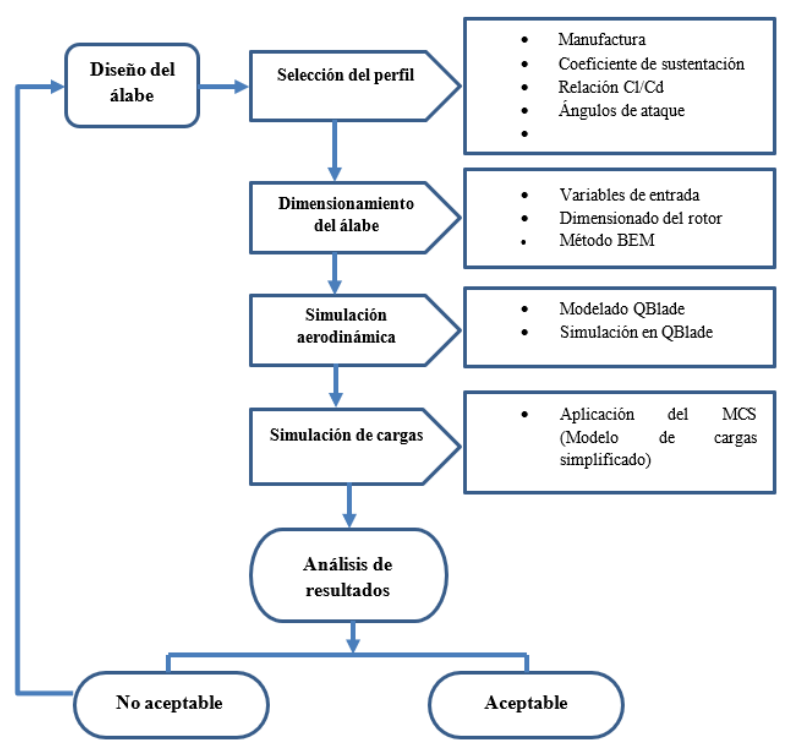

Figura 1 Metodología para el diseño del álabe

\section{Selección del perfil aerodinámico}

El elemento importante en el diseño de álabes es el perfil aerodinámico, el objetivo del perfil es generar una fuerza motriz a través de la fuerza de sustentación. No existe una metodología para seleccionar un perfil aerodinámico, pero se debe tomar en cuenta ciertas especificaciones y adecuarlas a las necesidades del diseño. Martínez presentó una propuesta para la selección de perfiles considerando varios criterios que ayudan a determinar el perfil óptimo (Martínez, 2007). Basados en estos criterios de selección, para la evaluación de los perfiles se consideraron los siguientes criterios: manufactura, coeficiente de sustentación, valor máximo $C_{L} / C_{D}$ y ángulo de ataque estable. Para el desarrollo del trabajo se preseleccionaron 4 perfiles aerodinámicos (A18, BW3, NACA4421 y NACA4424) que actualmente se utilizan para diseño de aerogeneradores de baja potencia (Carantoña, 2009). En la Figura 2 se muestran los perfiles seleccionados.

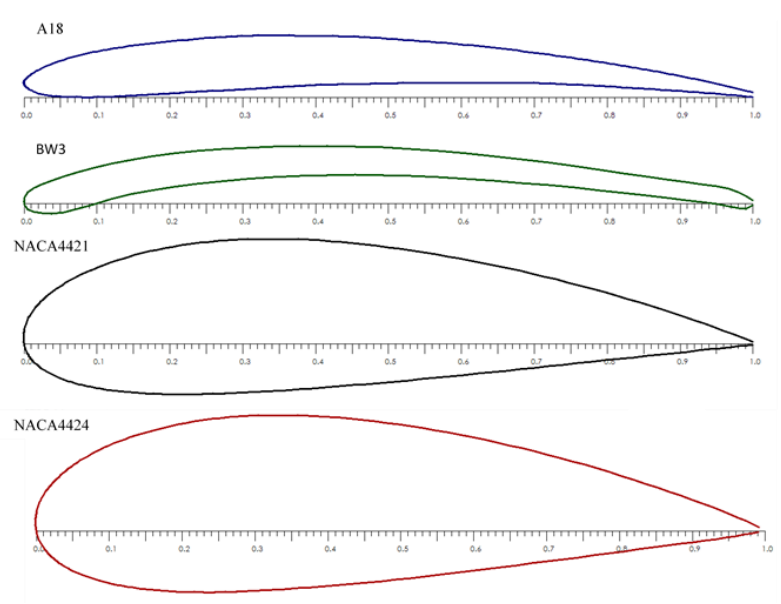

\section{Manufactura}

Para la manufactura de los álabes se evalúa la forma geométrica del perfil aerodinámico. Con el fin de encontrar un perfil con geometría simple ayude al proceso de maquinado. Para ello, se comparan sus propiedades geométricas tales como: mejor simetría, porcentaje de espesor mayor, porcentaje de curvatura menor y borde de salida robusto. La Figura 2 muestra la geometría de los perfiles seleccionados A18, BW3, NACA4421 y NACA4424.

De la Figura 2, se puede observar que los bordes de salida de los perfiles A18 y BW3 son más delgados en comparación con los perfiles NACA424 y NACA4421. Asimismo, se observa que los perfiles NACA tienen una geometría simétrica. En la tabla 1, se presentan los valores geométricos de cada perfil y con bases a esos valores se define el perfil NACA4424 como la mejor opción para el proceso de manufactura, este perfil tiene un porcentaje de espesor promedio de $26.60 \%$ mayor a los otros perfiles y su porcentaje de curvatura es el de menor valor con $22.94 \%$.

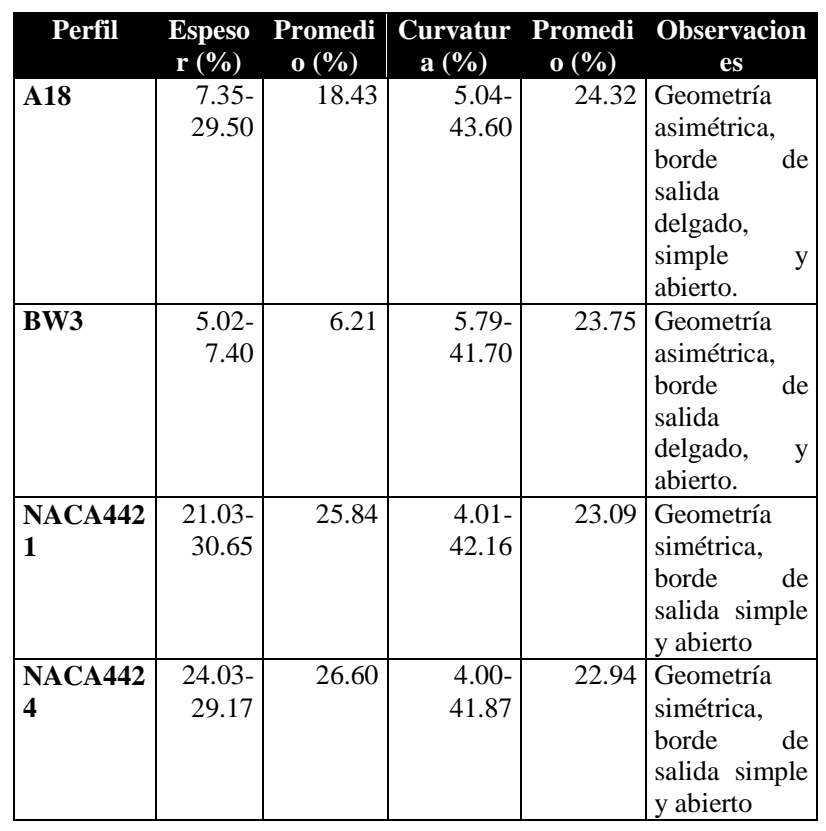

Tabla 1 Comparación de los perfiles aerodinámicos de acuerdo a su geometría

\section{Coeficiente de sustentación}

El valor del coeficiente de sustentación es directamente proporcional al ángulo de ataque, a mayor ángulo de ataque, se desarrolla una mayor diferencia de presiones y mayor impacto en el intradós del perfil, aumentando así la magnitud de la fuerza de sustentación. 
Este criterio se evalúa analizando la caída del coeficiente de sustentación, se selecciona al perfil que tenga una caída de sustentación de forma gradual (descenso $<25 \%$ ), si tiene una caída abrupta, es decir, tres grados antes del $\alpha_{\text {stall }}$ y un descenso mayor al $55 \%$ provoca fuertes vibraciones en los álabes (Pavón, 2019). Por esta razón, se recomienda que el descenso debe mantenerse en un rango entre 25 al $55 \%$ para que el sistema cuando entre en pérdida aerodinámica, no presente vibraciones y evite el embalamiento del rotor. En la Figura 3, región 2 , se muestra los diferentes comportamientos de la caída de sustentación.

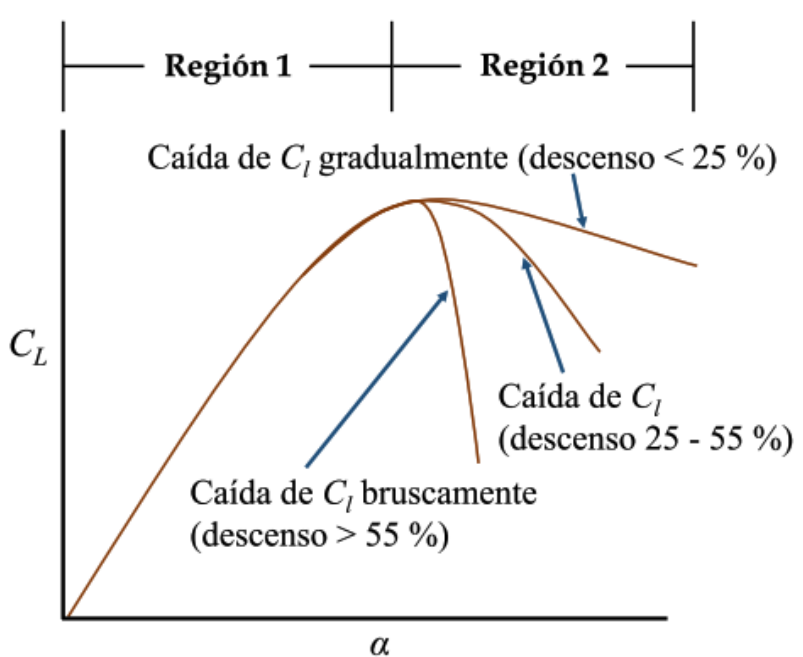

Figura 3 Comportamiento del coeficiente de sustentación

Para la evaluación del coeficiente de sustentación realiza una simulación con el software XFLR5, para obtener el comportamiento del coeficiente de sustentación de los perfiles aerodinámicos. Para esta simulación se considera un rango de 100,000 a 500,00 número de Reynolds (X. Tang, 2015). En la figura 4 se observan las gráficas de las curvas del $C_{L}$ en función de los ángulos de ataque.

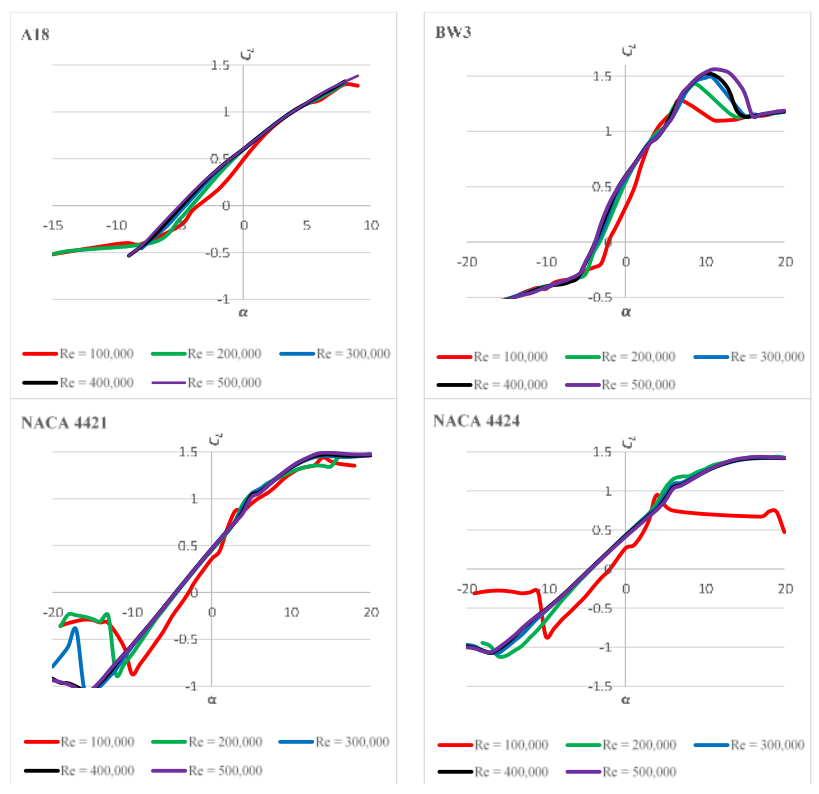

Figura 4 Curvas del $C_{L}$ de los perfiles aerodinámicos

En la Figura 4 se aprecia que los perfiles alcanzan la sustentación máxima en los 500,000 $R e$, mientras que la caída de sustentación se presenta en los 100,000 Re. Los valores del $C_{L}$ máx. en función al $\alpha$ de cada uno de los perfiles se resumen en la Tabla 2. Donde se determinó que el perfil con mayor sustentación es el BW3 con $C_{L}$ máx. $=1.55$, para un número $\mathrm{Re}$ de 500,000 y presenta una caída de sustentación en $\alpha_{\text {stall }}=11^{\circ}$.

\begin{tabular}{|l|r|r|r|r|r|} 
Perfil & \multicolumn{2}{c}{$\begin{array}{c}\text { Sustentación } \\
\text { máxima }\end{array}$} & \multicolumn{3}{c|}{$\begin{array}{c}\text { Caída de } \\
\text { sustentación }\end{array}$} \\
\cline { 2 - 6 } & $\begin{array}{l}\boldsymbol{C}_{\boldsymbol{L}} \\
\text { máx. }\end{array}$ & $\boldsymbol{R e}$ & $\boldsymbol{\alpha}$ & $\boldsymbol{R} \boldsymbol{}$ & $\boldsymbol{\alpha}_{\text {stall }}$ \\
\hline A18 & 1.38 & 500,000 & 9 & 100,000 & $7^{\circ}$ \\
\hline BW3 & 1.55 & 500,000 & 12 & 100,000 & $11^{\circ}$ \\
\hline NACA4421 & 1.49 & 500,000 & 14 & 100,000 & $15^{\circ}$ \\
\hline NACA4424 & 1.44 & 500,000 & 17 & 100,000 & $6^{\circ}$ \\
\hline
\end{tabular}

Tabla 2 Valores del CL máx. y caída de sustentación

\section{Valor máximo $\mathrm{C}_{\mathrm{L}} / \mathrm{C}_{\mathrm{D}}$}

La relación entre sustentación y arrastre es de gran importancia en la selección del perfil porque es posible determinar su eficiencia y el ángulo de ataque máximo en el que trabaja sin disminuir sustentación por efectos de desprendimiento de capa limite y de excesivo arrastre (Carantoña, 2009). En esta evaluación se obtiene el punto de mayor sustentación con menor arrastre. En la Figura 5, se muestra como es el comportamiento de la relación sustentación y arrastre de cada uno de los perfiles simulados con XFLR5. 


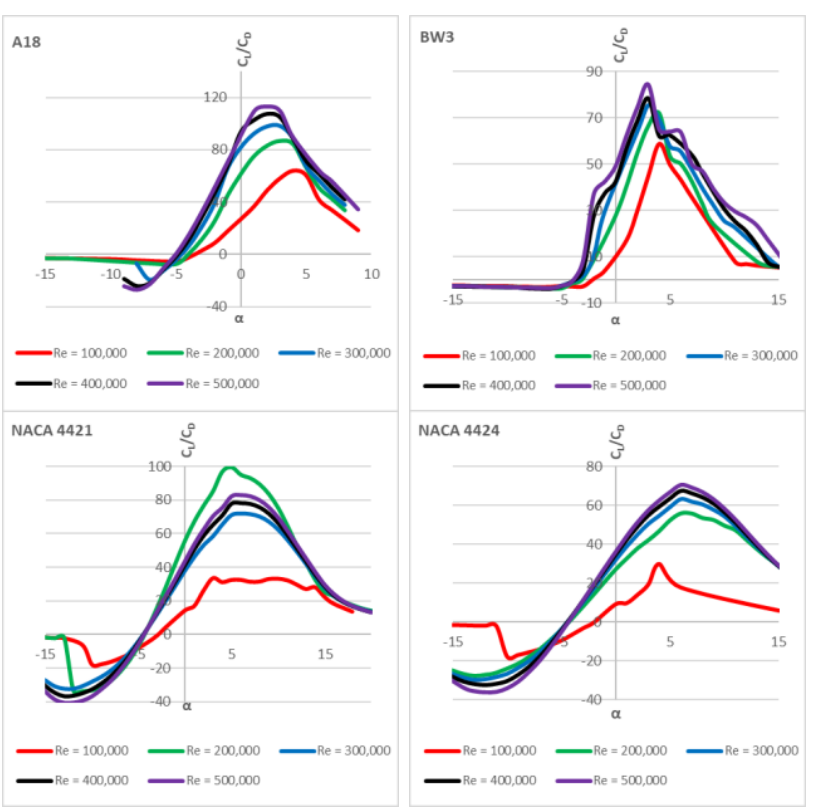

Figura 5 Curvas $C_{L} / C_{D}$ de los perfiles aerodinámicos

En la Figura 5 se observa que el perfil A18 obtiene la máxima relación $C_{L} / C_{D}=113$ en $\alpha=2^{\circ}$ con $\mathrm{Re}=500,000$. Mientras que el perfil NACA4421 obtiene la máxima relación $C_{L} / C_{D}=99.28$ en $\alpha=6^{\circ}$ y $\mathrm{Re}=200,00$. En la Tabla 3 se resumen los resultados de la relación $C_{L} / C_{D}$ de cada perfil, donde se observa que el A18 tiene mayor eficiencia.

\begin{tabular}{|c|c|c|c|}
\hline Perfil & Máx. relación & $\operatorname{Re}$ & $\alpha$ \\
\hline A18 & 113 & 500,000 & 2 \\
\hline BW3 & 84.43 & 500,000 & 3 \\
\hline NACA4421 & 99.28 & 200,000 & 6 \\
\hline NACA4424 & 70.66 & 500,000 & 6 \\
\hline
\end{tabular}

Tabla 4 Resultados de los parámetros máximos $C_{L} / C_{D}$

\section{Ángulo de ataque estable ( $\alpha$ )}

El coeficiente de sustentación depende del ángulo de ataque (véase Figura 3). A mayor ángulo de ataque, mayor sustentación, pero en la zona de pérdida aerodinámica (región 2 de Figura 3) la sustentación disminuye. Tomando en cuenta esa relación, el perfil debe mantener un rango de ángulos de ataque para que la caída de sustentación sea estable aún dentro de la zona de pérdida aerodinámica. En este criterio propone un rango de $4^{\circ}$ a $7^{\circ}$ (Martínez, 2007).

\section{Dimensionado del álabe}

El dimensionado del álabe es la aplicación del método BEM. Se basa en un proceso de iteraciones que distribuye la longitud de cuerda y el ángulo de torsión a través del radio total del rotor, con esto proceso se define la geometría del álabe.

\section{Variables de entrada}

Para iniciar este proceso, se consideran los criterios de diseño mencionados en la Tabla 1, a partir de esos valores se dimensiona el rotor y después se aplica el método BEM.

\begin{tabular}{|l|r|r|r|}
\hline \multicolumn{4}{|c|}{ Clase II SWT Norma IEC 61400-2 } \\
Descripción Valores & \multicolumn{1}{c|}{ Unidad } \\
\hline $\begin{array}{l}\text { Potencia nominal } \\
\text { del aerogenerador }\end{array}$ & $P_{n o m}$ & 5000 & $\mathrm{~W}$ \\
\hline Velocidad media & $V_{m}$ & 7.5 & $\mathrm{~m} / \mathrm{s}$ \\
\hline $\begin{array}{l}\text { Velocidad de } \\
\text { diseño }\end{array}$ & $V_{d}$ & 10.5 & $\mathrm{~m} / \mathrm{s}$ \\
\hline $\begin{array}{l}\text { Coeficiente de } \\
\text { potencia }\end{array}$ & $C_{p}$ & 0.4 & {$[-]$} \\
\hline Densidad del aire & $\rho$ & 1.225 & $\mathrm{~kg} / \mathrm{m}^{3}$ \\
\hline $\begin{array}{l}\text { Eficiencia del } \\
\text { aerogenerador }\end{array}$ & $\eta g$ & 0.8 & {$[-]$} \\
\hline $\begin{array}{l}\text { Eficiencia de la } \\
\text { caja } \\
\text { multiplicadora }\end{array}$ & $\eta i$ & 0.95 & {$[-]$} \\
\hline Área de barrido & & & \\
\hline Radio total & $A T$ & 23.200 & $\mathrm{~m}^{2}$ \\
\hline $\begin{array}{l}\text { No. de elementos } \\
\text { del álabe }\end{array}$ & $R T$ & 2.717 & $\mathrm{~m}$ \\
\hline $\begin{array}{l}\text { Colocación del } \\
\text { ler perfil= }\end{array}$ & $N e$ & & {$[-]$} \\
\hline Número de aspas & $I$ & & \\
\hline $\begin{array}{l}\text { Viscosidad } \\
\text { cinemática }\end{array}$ & $v$ & $1.551 \mathrm{E}-05$ & $\mathrm{~m}^{2} / \mathrm{s}$ \\
\hline $\begin{array}{l}\text { Perfil } \\
\text { aerodinámico }\end{array}$ & & & \\
\hline
\end{tabular}

Tabla 4 Variables de entrada para dimensionamiento del álabe

\section{Dimensionamiento del rotor}

El objetivo principal de los cálculos de dimensionamiento es determinar el área del rotor y el radio del álabe (Pavón, 2019). A continuación, se enlistan las ecuaciones para realizar este proceso.

Velocidad nominal

$V_{\text {nominal }}=1,4 V_{m}$

Potencia mecánica

$P_{\text {mec }}=\frac{1}{2} \rho A V_{\text {nominal }}^{3} C_{p}$.

Área de barrido del rotor

$A=\frac{2 P_{m e c}}{\rho V_{\text {nominal }}^{3} C p}$

Radio del álabe

$$
R=\sqrt{\frac{2 P_{\text {mec }}}{\frac{1}{2} \pi \rho V_{\text {nominal }}^{3}}} .
$$


Velocidad angular del rotor $\left(\Omega_{\text {rotor }}\right)$

$T_{m e c}=\frac{P_{m e c}}{\Omega_{\text {rotor }}}$.

Velocidad del álabe

$\lambda=\frac{\Omega r}{U_{1}}$.

\section{Método BEM (Blade Element Momentum)}

El proceso de interacción se realiza en una hoja de cálculos y se determinan todos los parámetros del método BEM, también, se realiza el número de interacciones necesarias hasta cumplirse las condiciones del método.

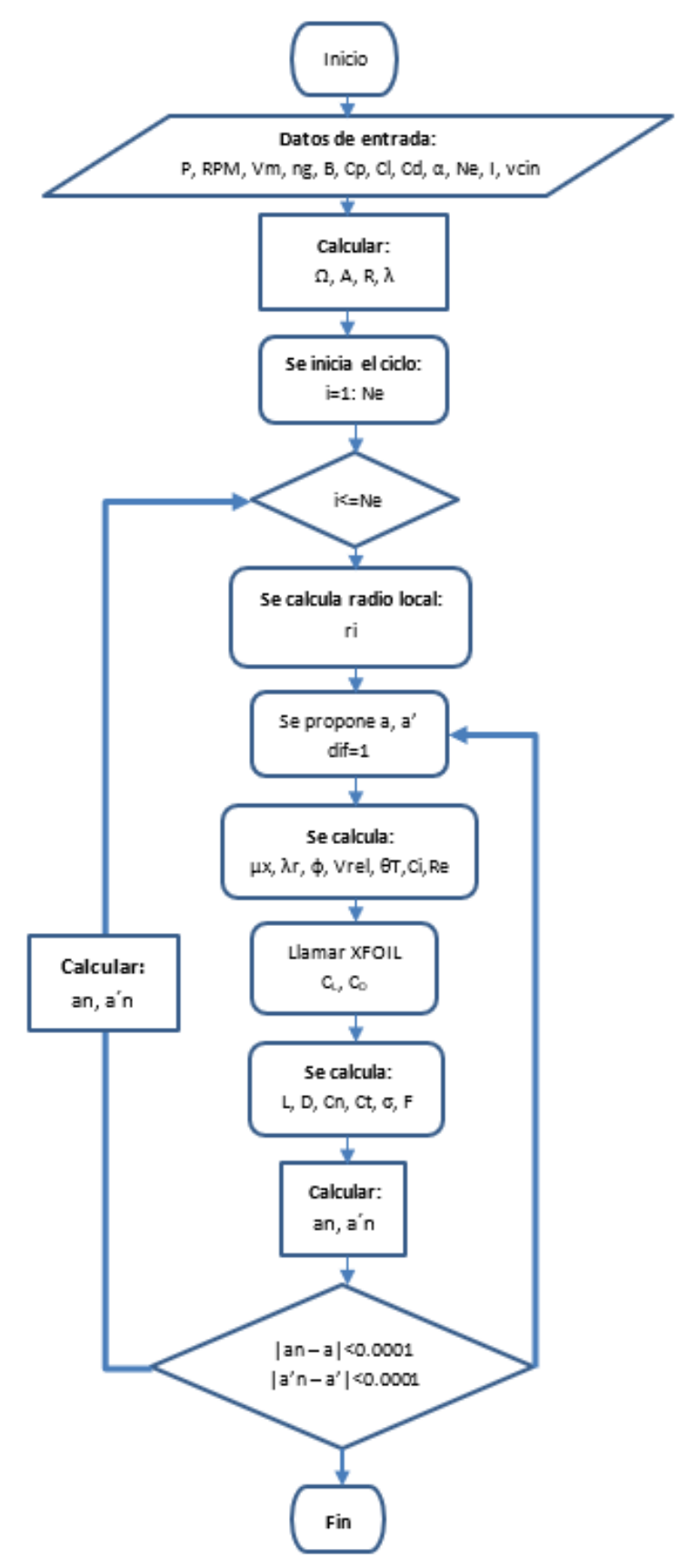

Figura 5 Diagrama de flujo propuesto para la aplicación del método BEM

\section{Simulación aerodinámica}

El análisis de la simulación estructural se basa en el estudio de cargas que se producen sobre el rotor de un aerogenerador de baja potencia. El análisis estructural se realiza con el software QBlade que está estructurado en dos módulos: diseño estructural del álabe y el análisis modal. El diseño estructural determina un modelo simple para un material isotrópico. Una vez determinado el modelo estructural, el software ejecuta un análisis modal. En la figura 7 se presenta el modelo de álabe diseñada en QBlade con los datos obtenidos del método BEM. Para el desarrollo del modelado se dividió por secciones y en cada sección se considera el ángulo torsión y la longitud de la cuerda.

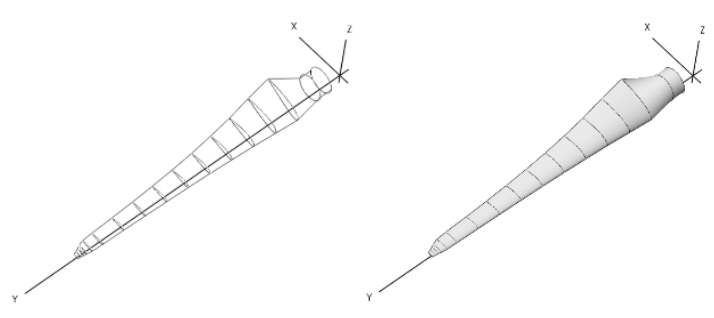

Figura 7 Modelado del álabe en QBlade

Los casos de carga a los que está sometido un álabe pueden modelarse como una viga en voladizo con una carga uniformemente distribuida. En las figuras 8 y 9, se muestran los dos tipos de vibraciones a los que se le somete el álabe a través de la simulación aerodinámica en QBlade. Una es de tipo Flapwise que se aprecia en la Figura 8 y en la Figura 9 se muestra la vibración tipo Edgewise. Esta simulación de QBlade muestra cómo se produce la flexión sobre el eje de la cuerda creando tensiones de compresión y tracción en la sección transversal del álabe.

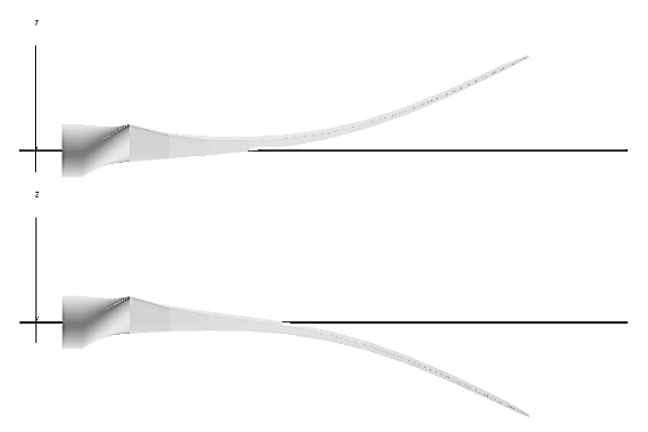

Figura 8 Vibración Flapwise 


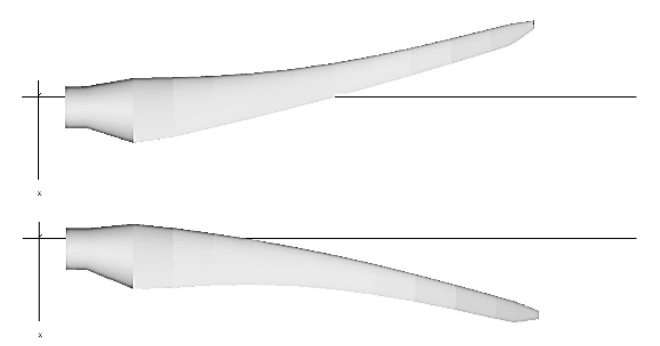

Figura 9 Vibración Edgewise

\section{Simulación de cargas}

Los casos carga simplificados que se aplican para la evaluación estructural del álabe se resumen en la Tabla 5. Las ecuaciones que se emplean, se enumeran a continuación de acuerdo al caso de carga especificado en la norma IEC 61400 .

\section{A: Cargas de fatiga en las aspas y el eje del rotor}

Cargas en el aspa:

$$
\begin{aligned}
\Delta F_{z B} & =2 m_{B} R_{\text {cog }} \Omega_{n, \text { diseño }}^{2}, \\
\Delta M_{x B} & =\frac{Q_{\text {diseño }}}{B}+2 m_{B} g R_{\text {cog }}, \\
\Delta M_{y B} & =\frac{\lambda_{\text {diseño }} Q_{\text {diseño }}}{B},
\end{aligned}
$$

Cargas en el eje:

$$
\begin{aligned}
& \Delta F_{x-e j e}=\frac{3}{2} \frac{\lambda_{\text {diseño }} Q_{\text {diseño }},}{R}, \\
& \Delta M_{x-e j e}=Q_{\text {diseño }}+\left(2 m_{r} g e_{r}\right), \\
& \Delta M_{e j e}=2 m_{r} g L_{r b}+\frac{R}{6} \Delta F_{x-e j e},
\end{aligned}
$$

\section{D: Empuje máximo en el eje}

$$
\Delta F_{x-e j e}=3.125 C_{T} \rho V_{m}^{2} \pi R^{2} .
$$

\section{E: Rotación máxima del viento}

Fuerza centrífuga en la raíz de la pala (eje z):

$$
F_{z B}=m_{B} \Omega_{n, \max }^{2} R_{\text {cog }} .
$$

Momento de flexión en el eje:

$$
M_{e j e}=m_{r} g L_{r b}+m_{r} e_{r} \Omega_{n, \max }^{2} L_{r b} \text {. }
$$

\section{F: Corto en la conexión de carga}

Momento de flexión en el eje:

$$
M_{x-\text { eje }}=G Q_{\text {diseño }} \text {. }
$$

Momento de flexión de la raíz de edgewise (eje $\mathrm{x})$ :

$$
M_{x B}=\frac{M_{x-e j e}}{B},
$$

\section{H: Carga extrema del viento}

Momento de flexión de la raíz flapwise (eje y):

$M_{y B}=C_{d} \frac{1}{4} \rho V_{e 50}^{2} A_{\text {proj,B}} R$,

Empuje máximo en el eje:

$M_{y B}=C_{l, \max } \frac{1}{6} \rho V_{e 50}^{2} A_{\text {proj }, B} R$.

Fuerza de empuje en el aspa:

$F_{x-e j e}=B C_{d} \frac{1}{2} \rho V_{e 50}^{2} A_{\text {proj,B }}$.

Fuerza de empuje en la torre:

$F_{x-e j e}=0,17 B A_{p r o j, B} \lambda_{e 50}^{2} \rho V_{e 50}^{2}$,

\section{I: Carga extrema del viento}

Cargar del aspa, nacelle y torre:

$F=C_{f} \frac{1}{2} \rho V_{e 50}^{2} A_{\text {proj }}$,

\section{Resultados}

\section{Selección del perfil aerodinámico}

De acuerdo los resultados de los criterios aplicados para la selección del perfil aerodinámico, se realiza una puntuación, en este caso asignando números del 4 al 1 , siendo 4 al que mejor se adapta al criterio y 1 al que menos cumple la propiedad. El propósito es promediar las puntuaciones y seleccionar el perfil con mayor eficiencia.

En la Tabla 5 se observa que el perfil que mejor cumple con los cuatro criterios es el NACA4421, siendo éste el que mejor satisface las características aerodinámicas. 


\begin{tabular}{|c|c|c|c|c|}
\hline $\begin{array}{l}\text { Criterios de } \\
\text { evaluación }\end{array}$ & A18 & BW3 & $\begin{array}{c}\text { Perfil } \\
\text { NACA4421 }\end{array}$ & NACA4424 \\
\hline Manufactura & 2 & 1 & 3 & 4 \\
\hline \begin{tabular}{|l|}
$\begin{array}{l}\text { Coeficiente de } \\
\text { sustentación }\end{array}$ \\
\end{tabular} & 1 & 4 & 3 & 2 \\
\hline Relación $\mathrm{C}_{\mathrm{L}} / \mathrm{C}_{\mathrm{D}}$ & 4 & 3 & 2 & 1 \\
\hline $\begin{array}{l}\text { Ángulos de } \\
\text { ataque estables }\end{array}$ & 2 & 3 & 4 & 1 \\
\hline Promedio & 2.25 & 2.75 & 3 & 2 \\
\hline
\end{tabular}

Tabla 5 Criterios de evaluación

\section{Dimensionado aplicando método BEM}

Aplicando el proceso iterativo BEM se determinaron los valores del radio, la cuerda y el ángulo de torsión que se usan para el dimensionado del aspa. Como se muestra en la Tabla 6 el álabe se dividió en 10 elementos, obteniendo una longitud total de $2.72 \mathrm{~m}$. Y en la Tabla 7 se presentan los valores de las fuerzas aerodinámicas del álabe obtenidas también del método BEM.

\begin{tabular}{|l|l|r|r|r|c|}
\hline \multicolumn{1}{|c|}{ Perfil } & Elemento & $\mathbf{r}[\mathbf{m}]$ & \multicolumn{1}{c|}{ cuerda $[\mathbf{m}]$} & $\boldsymbol{\beta}\left[^{\circ}\right]$ & Re \\
\hline Circular & 0 & 0.08 & 0.19 & & \\
\hline Circular & 0 & 0.27 & 0.19 & & \\
\hline NACA4421 & 1 & 0.41 & 0.33 & 26.45 & 438520.99 \\
\hline NACA4421 & 2 & 0.66 & 0.41 & 17.55 & 486486.68 \\
\hline NACA4421 & 3 & 0.92 & 0.33 & 12.19 & 503449.25 \\
\hline NACA4421 & 4 & 1.18 & 0.28 & 7.78 & 532080.73 \\
\hline NACA4421 & 5 & 1.43 & 0.24 & 7.51 & 536867.16 \\
\hline NACA4421 & 6 & 1.69 & 0.21 & 3.85 & 538621.16 \\
\hline NACA4421 & 7 & 1.95 & 0.18 & 2.60 & 535928.80 \\
\hline NACA4421 & 8 & 2.20 & 0.15 & 1.63 & 518527.38 \\
\hline NACA4421 & 9 & 2.46 & 0.12 & 0.84 & 448404.84 \\
\hline NACA4421 & 10 & 2.72 & 0.11 & 0.20 & 434152.00 \\
\hline
\end{tabular}

Tabla 6 Resultados finales del dimensionamiento del álabe

\begin{tabular}{|l|r|r|r|c|c|c|c|}
\hline \multicolumn{1}{|c|}{ Elemento } & \multicolumn{1}{c|}{$\mathbf{D}$} & $\mathbf{D}$ & $\mathbf{C n}$ & $\mathbf{C t}$ & $\boldsymbol{\sigma}$ & $\mathbf{a}$ & $\mathbf{a}$ \\
\hline $\mathbf{1}$ & 58.295 & 0.847 & 0.882 & 0.543 & 0.589 & 0.311 & 0.215 \\
\hline $\mathbf{2}$ & 86.875 & 1.235 & 0.949 & 0.398 & 0.297 & 0.306 & 0.088 \\
\hline $\mathbf{3}$ & 115.295 & 1.631 & 0.979 & 0.306 & 0.172 & 0.302 & 0.047 \\
\hline $\mathbf{4}$ & 154.463 & 1.829 & 1.028 & 0.239 & 0.116 & 0.343 & 0.031 \\
\hline $\mathbf{5}$ & 186.192 & 2.200 & 1.036 & 0.198 & 0.080 & 0.343 & 0.021 \\
\hline $\mathbf{6}$ & 217.840 & 2.572 & 1.041 & 0.168 & 0.058 & 0.343 & 0.015 \\
\hline $\mathbf{7}$ & 247.904 & 2.928 & 1.045 & 0.145 & 0.044 & 0.343 & 0.011 \\
\hline $\mathbf{8}$ & 270.364 & 3.219 & 1.048 & 0.128 & 0.034 & 0.343 & 0.009 \\
\hline $\mathbf{9}$ & 261.636 & 3.252 & 1.056 & 0.113 & 0.023 & 0.344 & 0.007 \\
\hline $\mathbf{1 0}$ & 279.775 & 3.514 & 1.060 & 0.102 & 0.019 & 0.345 & 0.006 \\
\hline
\end{tabular}

Tabla 7 Fuerzas aerodinámicas del álabe

\section{Simulación en QBlade}

En esta sección se muestran los resultados de la simulación realizada en QBlade. A continuación, se describen los resultados de la simulación del software Qblade. El software contiene módulos de simulación basado en el método BEM.
En la figura 10 se muestran las curvas de potencia que el aerogenerador puede entregar con el modelo del álabe diseñada. En este caso, la simulación está en función de la velocidad del viento en un rango de 1 a $25 \mathrm{~m} / \mathrm{s}$. Asimismo, se presenta la variación de la velocidad de giro, y se considera un ángulo de paso de $0^{\circ}$.

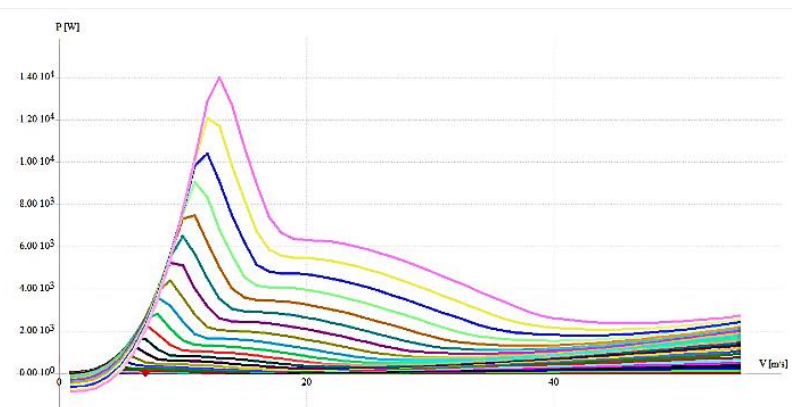

Figura 10 Curvas de potencia en relación a la velocidad del viento

En la Figura 10, se observa que el aerogenerador puede entregar más de $5 \mathrm{~kW}$ con el diseño del álabe que se obtuvo.

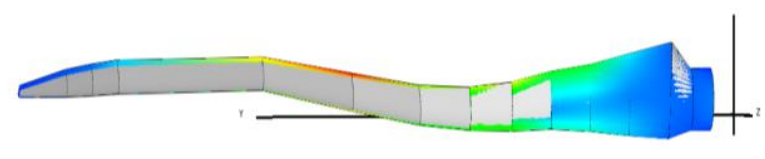

Figura 11 Distribución de las deflexiones ocasionadas por las cargas

En la Figura 11 se muestra la distribución de deflexiones ocasionadas por las cargas a la que se sometió durante la simulación. Y en la tabla 8 se muestra los esfuerzos máximos obtenidos con la simulación estructural.

\begin{tabular}{|l|r|}
\hline \multicolumn{1}{|c|}{ Radio del aspa } & Máx. esfuerzo por sección [Pa] \\
\hline 0.08 & 17707800 \\
\hline 0.27 & 14676600 \\
\hline 0.41 & 11046200 \\
\hline 0.66 & 11050400 \\
\hline 0.92 & 6127820 \\
\hline 1.18 & 6351670 \\
\hline 1.43 & 10554700 \\
\hline 1.69 & 11735600 \\
\hline 1.95 & 6846020 \\
\hline 2.20 & 15093300 \\
\hline 2.46 & 17090700 \\
\hline 2.72 & 12472800 \\
\hline 0.08 & 12537000 \\
\hline 0.27 & 10790300 \\
\hline 0.41 & 10697100 \\
\hline 0.66 & 7207970 \\
\hline 0.92 & 0 \\
\hline
\end{tabular}

Tabla 8 Evaluación del álabe por MCS 


\section{Resultados del MCS}

En la Tabla 9 se presentan los resultados obtenidos del cálculo del modelo de cargas simplificados, donde se obtienen los valores para cada caso de carga en este estudio. En el caso de carga $\mathrm{A}$ es un análisis de fatiga, donde se muestran las cargas en el aspa y en el eje, y el valor máximo de fatiga es la fuerza centrífuga en la raíz del álabe con un valor de $21421.6626 \mathrm{~N}$. Para el caso del análisis de resistencia a la ruptura se presenta con mayor relevancia el caso de carga $\mathrm{H}$, y es la fuerza de empuje sobre el eje con un valor de 10573.4736 N. Por otro lado, los valores máximos de momento para cada sentido del aspa son: en el sentido flapwise es de 698.4127 Nm, y en el sentido edgewise es de 425.5041 Nm. Para fines de ensayos, el álabe está sometida a cargas, en este contexto, el momento máximo se presenta en el sentido flapwise. Dicho momento debe resistir la raíz del álabe, que es donde está unida al buje del aerogenerador. Es importante mencionar, que este modelo ofrece un diseño robusto brindando mayor seguridad al sistema.

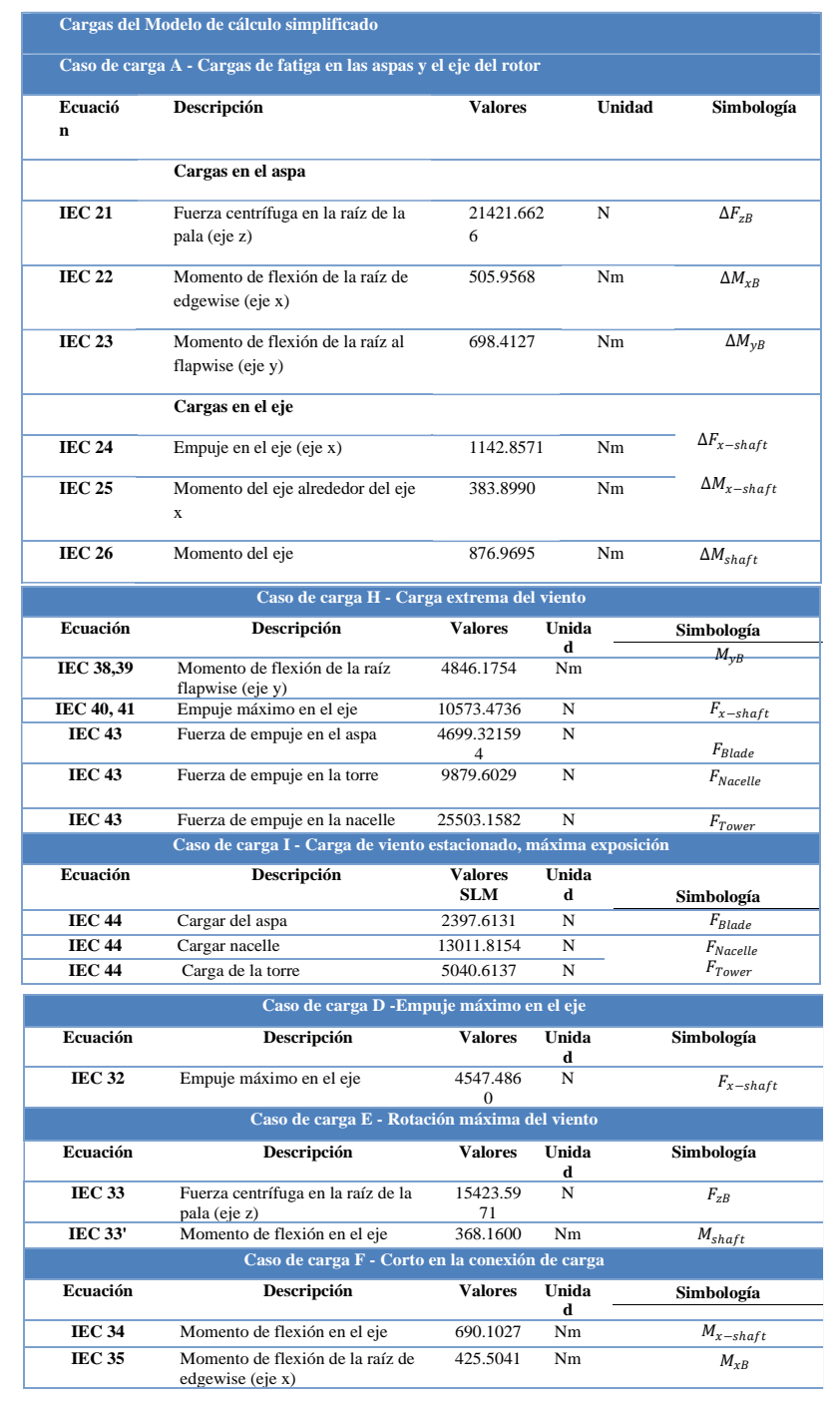

Tabla 5 Casos de carga de acuerdo a la norma IEC 61400

\section{Agradecimientos}

Los autores desean agradecer al Mtro. José Rafael Dorrego Portela, de la Universidad del Itsmo, campus Tehuantepec, por su asesoría en el uso de los softwares XFLR5 y QBlade.

\section{Conclusiones}

En primera instancia se concluye que la metodología para la selección de perfiles aerodinámicos de acuerdo a los criterios establecidos, es aceptable. Con el objetivo de seleccionar el perfil óptimo por medio de una evaluación de alternativas, se eligió el perfil NACA4421.

El dimensionado del álabe consistió en desarrollar la metodología mediante el método BEM. Se puede destacar que es necesario datos como: la velocidad de viento, coeficientes aerodinámicos del perfil y potencia del aerogenerador. Además, con resultados de la distribución de la cuerda y torsión se logra generar el modelo en QBlade.

El estudio del análisis aerodinámico permitió garantizar que la geometría del álabe es apta para un aerogenerador de $5 \mathrm{~kW}$ de potencia nominal. Referente al análisis estructural se recomienda aplicar modelo de carga simplificado (MCS), donde permite obtener las fuerzas y momentos máximos. Así, determinar un diseño preliminar del aerogenerador, este método se basa en la norma IEC 61400-2.

Con los resultados presentados en el trabajo, se concluye que el modelo de álabe cumple con la eficiencia aerodinámica y con el límite de daño por fatiga, por lo tanto, garantiza su buen funcionamiento.

\section{Referencias}

Carantoña, O. (2009). Análisis del comportamiento aerodinámico de perfiles empleados en aerogeneradores de baja potencia. Fuentes Alternas de Energía y Generación Distribuida.

International Electrotechnical Comission. (2013). IEC 61400-2 Wind turbines- Part 2: Small wind turbines. Switzerland. 
International Electrotechnical Comission. (2014). IEC 61400-2 Wind turbines- Part 23: Full-scale structural testing of rotor blades. Switzerland.

Martínez, J. (2007). Diseño, Manufactura y Caracterización Experimental de Aspas y Controlador de Carga Resistiva para una Turbina de Viento Bergey BWC XLI-Edición única. Tecnológico de Monterrey.

Pavón, G. V. (2019). Modelación de respuesta aerodinámica para aspas con stall pasivo-Tesis de Maestría. Santo Domingo Tehuantepec, Oaxaca: Universidad del Istmo.

X. Tang, X. H. (2015). A Direct Approach of Design Optimization for Small Horizontal Axis Wind Turbine Blades. Procedia CIRP, vol. 36, 12-16. 